\title{
(Politik-)didaktische Handlungsempfehlungen
}

Der arbeitsweltorientierte Zugang zu politischer Bildung orientiert sich an den subjektiven Vorstellungen der Lernenden über die Arbeitswelt und hat Politisches Lernen zum Gegenstand. Um diesen Zugang zu politischer Bildung zu fördern, bedarf es empirisch fundierter Erkenntnisse. Die Ergebnisse der vorliegenden quantitativen und qualitativen Studie zu den Vorstellungen über die Arbeitswelt der Jugendlichen am Übergang von der Sekundarstufe I in die Sekundarstufe II sollen mit der Forschungsfrage „Was brauchen Jugendliche in Bezug auf Berufsorientierung von außen betrachtet und welche Angebote sollten sie erhalten?" aus einer subjektorientierten Bildungsperspektive analysiert werden und im Sinne eines (politik-)didaktischen rekonstruktiven Verfahrens (vgl. Lange 2007, 58-65) zu konkreten Handlungsempfehlungen führen. Die didaktische Strukturierung der Studienergebnisse wird als Zielklärung für didaktische Handlungsempfehlungen gesehen. Dabei wird der Frage nachgegangen, ,[w]elchen Beitrag [...] die untersuchten fachlichen Vorstellungen zu einer demokratischen Bürgerschaftsbildung [liefern]“" (ebd., 63).

Individuelle Berufsentscheidungen sind sowohl mit der eigenen sozialen Herkunft, dem sozialen Geschlecht, Schulnoten etc. verbunden und hängen gleichzeitig auch von den gesellschaftlichen Strukturen, wie Ausbildungs- und Arbeitsmarkt, finanzieller Unterstützung während der Ausbildung und Studienplatzangebot ab (vgl. Abschn. 2.1 Entwicklung der beruflichen Biografie im Spannungsfeld zwischen Individuum und Gesellschaft, 8). Die subjektorientierte politische Bildung mit dem Schwerpunkt Arbeitsweltorientierung setzt hier beim Individuum an und sieht Berufsorientierung als wichtigen Aspekt einer demokratischen Gesellschaft. Entscheidungen über die Berufswahl sind ausschlaggebend für die aktive Teilhabe in der Gesellschaft. Ziel der Berufsorientierung ist somit auch die Bildung mündiger, selbstbestimmter Bürger*innen unter der Berücksichtigung der unterschiedlichen Ausgangspositionen, wie soziale Herkunft, Gender 
oder Migrationshintergrund. Hier steht nicht die Passung auf dem Arbeitsmarkt im Vordergrund, sondern die Entfaltung des beruflichen Selbstkonzeptes der Jugendlichen (vgl. Gottfredson 1981, 1996). Daran anknüpfend wird das Individuum in den Mittelpunkt der didaktischen Überlegungen gestellt.

Wie die 2011 von der Arbeiterkammer in Auftrag gegebene Jugendwertestudie (Heinzlmaier/Ikrath: 2011) zeigt, steht für die Jugendlichen am Ende der Sek I ein sicherer Arbeitsplatz auf dem ersten Platz. Auch die vorliegende quantitative Studie hat ergeben, dass ein sicherer Arbeitsplatz ganz oben auf der Liste steht, wenn es um die eigenen Bedürfnisse in der Arbeitswelt geht. Daran anknüpfend sollte die didaktische Strukturierung der Bildungsinterventionen das individuelle Bedürfnis nach einem sicheren Arbeitsplatz berücksichtigen. Dazu gehört, dass das nötige Wissen über die aktuellen Entwicklungen des Arbeitsmarktes vermittelt wird und gleichzeitig ist der sichere Arbeitsplatz auch als ein Punkt auf der politischen Agenda zur Sicherung von Arbeitsplätzen und zur fortlaufenden Förderung von Bildungsangeboten zu sehen.

Anhand der empirischen Ergebnisse wurden verschiedene didaktische Handlungsempfehlungen abgeleitet. Deren Ziel ist es, Jugendliche besser in ihrer Bildungs- bzw. Berufsentscheidung zu unterstützen. Im Folgenden werden die einzelnen Handlungsempfehlungen im Detail erläutert.

\section{Erweiterung der schulischen Berufsorientierungsangebote und Elternbildung}

Grundsätzlich lässt sich ein Spannungsfeld zwischen privater Berufsorientierung durch die Eltern und das soziale Umfeld und öffentlicher Berufsorientierung, welche in der Regel in oder durch die Schule stattfindet, feststellen. Die empirischen Ergebnisse haben gezeigt, welche enorme Wirkung die Eltern auf die Jugendlichen haben (vgl. Abschn. 3.4.3.2 Einfluss auf Berufs- und Bildungsentscheidungen, 141). Deshalb ist es zentral, bei den Eltern das Bewusstsein dafür zu bilden, welchen großen Einfluss sie haben. Dies kann zum Beispiel durch Workshops für Eltern, deren Kinder sich in dieser wichtigen Entscheidungsphase befinden, geschehen.

Vor allem mit Blick auf bildungsferne Jugendliche und Jugendliche mit Migrationshintergrund sollte geprüft werden, welche Unterstützungsangebote eine eventuelle fehlende oder unzureichende Unterstützung durch ihre Eltern auffangen würden. Denkbar wären hier veränderte schulische Orientierungsangebote, wie beispielsweise Mentor*innenprogramme, die durch eine zusätzliche Unterstützung den Einstieg in Berufsthemen leichter machen.

Es ist zu betonen, dass die Eltern zwar eine große Rolle spielen, es jedoch nicht in ihrer Verantwortung allein liegen sollte, Bildungsdefizite auszugleichen. 
Die Unterstützung sollte vielmehr, im Sinne der Chancengleichheit, im öffentlichen Bildungsauftrag verankert sein, damit sie für jede*n Jugendliche*n zugänglich ist. Ungleichheiten der sozialen Herkunft können besser ausgeglichen werden, wenn Chancengleichheit durch formale Berufsorientierung in der Schule geschaffen wird. Dies kann zum Beispiel durch den Unterricht „Berufsorientierung“, innerhalb dessen auch wirtschaftsdemografische Themen behandelt werden, geschehen, ggfs. mit Einbindung der Eltern.

Die Studie hat gezeigt, dass der Besuch eines „Tags der offenen Tür“ von den Schüler*innen als wichtig bei der Entscheidung für eine weiterführende Schule wahrgenommen und dass dieser Besuch meist durch die Eltern organisiert wird. Soziale Ungleichheiten können somit auch dadurch abgeschwächt werden, indem Besuche zu verschiedenen „Tagen der Offenen Tür“" von der Schule organisiert werden und nicht nur durch private Initiativen der Eltern. Dadurch kommt es hierbei zu einer Minimierung der Abhängigkeit von Eltern und anderen privaten Bezugspersonen und alle Jugendlichen werden im selben Maße informiert.

Da das soziale Umfeld und der Beruf der Eltern sehr ausschlaggebend für die Bildungs- und Berufsentscheidung der Jugendlichen ist und auch die berufspraktischen Tage mehrheitlich in elterlichen Betrieben oder Unternehmen aus dem Bekanntenkreis der Eltern absolviert werden (vgl. Abschn. 3.4.3.2 Einfluss auf Berufs- und Bildungsentscheidungen, 141), sollten die flächendeckenden berufspraktische Tage für alle Achtklässler*innen in Österreich nicht unbedingt im eigenen sozialen Umfeld stattfinden. Der Vorschlag zur Umgestaltung der berufspraktischen Tage sieht vor, dass hierfür alle Schnuppermöglichkeiten aus dem Bekanntenkreis der Jugendlichen im selben Jahrgang auf einer Liste gesammelt werden und neu unter den Schüler*innen verteilt werden. Jede*r Schüler*in nennt eine Praktikumsstelle, wobei er*sie einen anderen Praktikumsplatz als den eignen Vorschlag wählen kann. Daraus ergibt sich die Möglichkeit, neue Berufe kennenzulernen bzw. auszuprobieren und trotzdem eine persönliche Betreuung bei den berufspraktischen Tagen durch die Eltern und Bekannten der Mitschüler*innen zu gewährleisten.

\section{Intensivere Berufsorientierung im Gymnasium}

In Abschn. 3.4.3.1 Allgemeine Vorstellungen über die Arbeitswelt (S. 139) zeigt sich, dass sich AHS-Schüler*innen tendenziell weniger informiert fühlen und sich deshalb oftmals für den Verbleib im Gymnasium entscheiden, um mehr Zeit für die Berufsentscheidung zu haben. Deshalb ist eine intensivere Berufsorientierung in den Gymnasien empfehlenswert, damit Jugendliche Berufsentscheidungen in der 4. 
Klasse Sek I informierter treffen können. Darunter fällt zum Beispiel, dass Jugendlichen die Möglichkeit gegeben wird, sich bewusst für ein Studium zu entschieden bzw. ein anderer Beruf- bzw. Bildungsweg in Erwägung gezogen werden kann.

\section{Reflektierende Berufsorientierung mit Fokus auf Gendergerechtigkeit}

Des Weiteren lässt sich aus den Ergebnissen des Abschn. 3.4.3.1 Allgemeine Vorstellungen über die Arbeitswelt (S. 139) ableiten, dass es Unterschiede zwischen den Geschlechtern gibt, mit Berufs- bzw. Bildungsentscheidung umzugehen. Mädchen wirken reflektierter im Abwägen der verschiedenen Faktoren ihrer Entscheidung, indem sie die Folgen dieser genauer hinterfragen. Damit verbunden weisen sie auch eine größere Unsicherheit in Bezug auf die eigenen Berufsentscheidungen auf. Ziel sollte sein, diese Erkenntnis als Chance für mehr Gendergerechtigkeit zu sehen und für alle Geschlechter eine reflektierende Berufsorientierung anzubieten. Daraus ergibt sich eine didaktische Handlungsempfehlung bestehend aus drei Schritten. Im ersten Schritt sollen die Jugendlichen im Rahmen des Berufsorientierungsunterrichtes angehalten werden, zu notieren, welche Faktoren ihnen im Beruf wichtig sind. Dadurch kommt es zu einer Reflexion der zentralen Punkte, die der zukünftige Job erfüllen soll. Daraufhin kann im zweiten Schritt eine Pro- und Kontra-Liste über die Wunschberufe verfasst werden, durch welche die Schüler*innen festhalten, welche Annahmen sie hinsichtlich ihrer Wunschberufe haben. Auch dieser Schritt dient der Reflexion. Im letzten Schritt sollen die Ergebnisse des ersten und zweiten Schrittes mit der Lehrkraft besprochen und Raum für Diskussion geschaffen werden. Dadurch können eventuelle Unsicherheiten oder fehlende Informationen festgestellt und gegebenenfalls relativiert werden. Somit kommt es zu einer Unterstützung der Jugendlichen, eine (selbst)bewusste und reflektierte Berufs- bzw. Bildungsentscheidung zu treffen. Trotz des Ziels, vor allem Mädchen in ihrer Zukunftsentscheidung zu stärken, profitieren auch Burschen in gleichem Maße von einem reflektierten Zugang zur Berufsorientierung.

Aus didaktischer Sicht sind vor allem Ansätze zu empfehlen, die auf inhaltliche Gemeinsamkeiten bei den Berufswünschen von Mädchen und Burschen rekurrieren. Die Ergebnisse der Studie haben inhaltliche Berufsbereiche aufgezeigt, für die sich beide Geschlechter in ähnlicher Weise zu interessieren scheinen (die untersuchendforschenden, künstlerisch-kreativen und ordnend-verwaltenden Berufe) und stellen einen interessanten Ansatzpunkt für die Ausrichtung der Berufsorientierung dar. Darüber hinaus stellt die Frage, nach Lebensentwürfen und Rollenvereinbarkeiten einen wichtigen Ausgangspunkt für gendergerechte Berufsorientierung dar. 


\section{Befähigung und Selbstwirksamkeit}

Die Literatur (vgl. Abschn. 2.4.1 Soziale Herkunft, 46) darauf hin, dass insbesondere die Dimension der Selbstwirksamkeit für Jugendliche vor allem im Zusammenhang mit sozialer Herkunft von Bedeutung ist. Die Ergebnisse der vorliegenden Studie haben gezeigt, dass die Jugendlichen die verschiedenen Berufe und Bildungsoptionen vor allem durch eine intensive Eigenrecherche im Internet kennenlernen, um so eine Berufsentscheidung zu treffen oder sich ein Bild über einen Wunschberuf machen zu können. Allerdings ergeben sowohl die qualitative als auch die quantitative Studie, dass die Schüler*innen oftmals nicht genau wissen, wo und wie sie sich ausreichend über Berufs- und Bildungswege informieren können (vgl. Abschn. 3.4.3.3 Unterstützung bei Berufs- und Bildungsentscheidungen, 145). Somit zeigen die Ergebnisse, dass die Fähigkeit, sich eigenmächtig relevante Informationen mittels Internet und Medien zu besorgen, bei den befragten Jugendlichen noch ausbaufähig ist. Die Jugendlichen sollen befähigt werden, ihre Eigenrecherche zu professionalisieren und zu hinterfragen. Im Zentrum steht dabei die Förderung von Medien- und Recherchekompetenzen. In der didaktischen Umsetzung sollte aber auch auf die Wahlfreiheit, die Interessen und Talente der Jugendlichen Bezug genommen werden, um eine Selbstwirksamkeit zu fördern. Weiters spielt in der Berufsorientierung ein breit angelegtes Faktenwissen über Berufe weiterhin eine wichtige Rolle. Der Umgang mit Medien und die Einschätzung bzw. Beurteilung des Informationsgehalts sind für zielführende Recherchekompetenzen ausschlaggebend. Demnach ist es wichtig, wie die Jugendlichen mit Medien umgehen und diese bewerten. Um sie dabei zu unterstützen, können zum Beispiel Erklärvideos mit Live-Aufnahmen aus dem Berufsalltag erstellt werden. Diese können dann im Schulunterricht angesehen sowie reflektiert oder aber auch zu Hause mit Reflexionsfragen bearbeitet werden. Diese Handlungsempfehlung basiert auf der Theorie Hollands (1996 - vgl. Abschn. 2.1.2 Berufswahl als Matching-Prozess, S. 11), welche besagt, dass die Passung zwischen individuellen Eigenschaften bzw. Fähigkeiten und der Berufswahl zentral ist, damit eine hohe Zufriedenheit mit der Arbeit entstehen kann. Auch die vorliegende Studie hat gezeigt, dass Jugendliche zwar oftmals Berufe, die zu ihren Interessen passen, in Betracht ziehen, es ihnen jedoch oftmals an Einblicken in den Beruf fehlt und sie deshalb dazu tendieren, einen Beruf zu wählen, in den sie durch ihr soziales Umfeld, meist durch die Eltern, bereits einen Einblick hatten. Mentor*innenprogramme im Hinblick auf Role Models und einen Einblick in unterschiedliche Lebenswelten dient hier beispielsweise einer Förderung von Jugendlichen über einen fachdidaktischen Zugang des Stärkens von Stärken bzw. von Interessen. 


\section{Berufsorientierung als Inhalt der Lehrer*innenbildung}

Ebenso stellt die Lehrer*innenbildung einen wichtigen Aspekt der Berufsorientierung dar. So weisen die Ergebnisse (vgl. Abschn. 3.4.3.2 Einfluss auf Berufsund Bildungsentscheidungen, 141) darauf hin, dass auch das Lehrpersonal die Jugendlichen in ihrer Berufs- bzw. Bildungsentscheidung beeinflusst. Dies geschieht einerseits, indem die Lehrer*innen als Informationsquelle dienen, und andererseits eine Vorbildwirkung einnehmen können. Deshalb soll eine Sensibilisierung für die Wichtigkeit der Unterstützung von Jugendlichen bei der Berufsorientierung einen integralen Bestandteil der Ausbildung von Lehrer*innen darstellen.

\section{Praxisorientierte Berufsorientierung}

Die Forschungsfrage, was Jugendliche brauchen, um eine Berufsentscheidung zu treffen, wurde von vielen Befragten mit dem Hinweis auf Praxiserfahrung und das Kennenlernen von möglichst vielen Berufen beantwortet. Daraus lässt sich aus didaktischer Sicht eine erfahrungsorientierte Berufsorientierung ableiten (vgl. Abschn. 3.4.3.3 Unterstützung bei Berufs- und Bildungsentscheidungen, 145). Um dies den Schüler*innen zu ermöglichen, können zum Beispiel Simulationsspiele oder Rollenspiele dazu dienen, die verschiedenen Berufe erlebbar zu machen. Auch eine Erweiterung der berufspraktischen Tage, vor allem in den Gymnasien, kann zu diesem Ziel beitragen. Allgemein sollte hier ein Schwerpunkt auf die Aneignung von Erfahrungswissen gelegt werden und Einblicke in Berufsbilder gegeben werden, die sich im Alltag nicht sonst nicht ergeben.

\section{Emotionale Unterstützung}

Sowohl innerhalb der Eltern- als auch der Lehrer*innenbildung ist die Auseinandersetzung mit den Ängsten und Unsicherheiten der Jugendlichen in Bezug auf ihre Bildungs- und Berufsentscheidung von zentraler Bedeutung. Wie die Ergebnisse zeigen, wünschen sich die befragten Jugendlichen eine emotionale Begleitung auf dem Weg in das Berufsleben und dass ihnen Zukunftsängste genommen werden. Darüber hinaus wünschen sie sich auch das bessere Kennenlernen ihrer individuellen Stärken (vgl. Abschn. 3.3.3.7 Was brauchen die Jugendlichen bzw. welche Wünsche haben sie in Bezug auf die bevorstehende Arbeitswelt?, 108). Auch in der Literatur (vgl. Abschn. 2.2.1 Österreich, 15) zeigt sich, dass Bildung und Ausbildung für die Jugendlichen wichtige Themen sind und gleichzeitig nicht frei von Sorgen. So besteht die größte Zukunftsangst der 11- bis 18-Jährigen in Österreich darin, später keinen Arbeitsplatz zu finden oder arbeitslos zu werden.

Selbsterfahrung und Reflexion werden deshalb als wichtige Bestandteile der Ausbildung für Lehrpersonen gesehen, um den Aufgaben und Herausforderungen der Berufsorientierung von Jugendlichen gewachsen zu sein. 
Open Access Dieses Kapitel wird unter der Creative Commons Namensnennung 4.0 International Lizenz (http://creativecommons.org/licenses/by/4.0/deed.de) veröffentlicht, welche die Nutzung, Vervielfältigung, Bearbeitung, Verbreitung und Wiedergabe in jeglichem Medium und Format erlaubt, sofern Sie den/die ursprünglichen Autor(en) und die Quelle ordnungsgemäß nennen, einen Link zur Creative Commons Lizenz beifügen und angeben, ob Änderungen vorgenommen wurden.

Die in diesem Kapitel enthaltenen Bilder und sonstiges Drittmaterial unterliegen ebenfalls der genannten Creative Commons Lizenz, sofern sich aus der Abbildungslegende nichts anderes ergibt. Sofern das betreffende Material nicht unter der genannten Creative Commons Lizenz steht und die betreffende Handlung nicht nach gesetzlichen Vorschriften erlaubt ist, ist für die oben aufgeführten Weiterverwendungen des Materials die Einwilligung des jeweiligen Rechteinhabers einzuholen. 